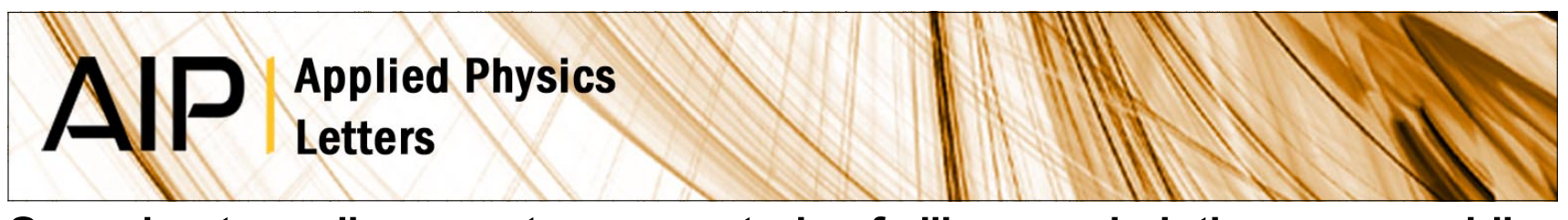

\title{
Scanning tunneling spectroscopy study of silicon and platinum assemblies in an opal matrix
}

C. Díaz-Guerra, J. Piqueras, V. G. Golubev, D. A. Kurdyukov, A. B. Pevtsov et al.

Citation: Appl. Phys. Lett. 77, 3194 (2000); doi: 10.1063/1.1325387

View online: http://dx.doi.org/10.1063/1.1325387

View Table of Contents: http://apl.aip.org/resource/1/APPLAB/v77/i20

Published by the AIP Publishing LLC.

\section{Additional information on Appl. Phys. Lett.}

Journal Homepage: http://apl.aip.org/

Journal Information: http://apl.aip.org/about/about_the_journal

Top downloads: http://apl.aip.org/features/most_downloaded

Information for Authors: http://apl.aip.org/authors

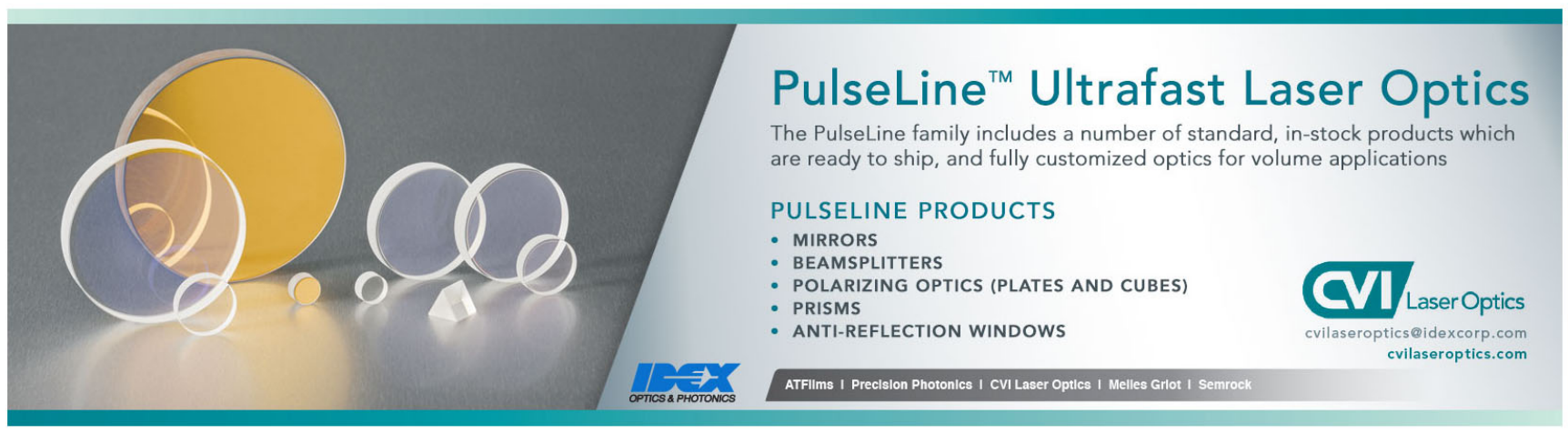




\title{
Scanning tunneling spectroscopy study of silicon and platinum assemblies in an opal matrix
}

\author{
C. Díaz-Guerra and J. Piqueras ${ }^{a}$ ) \\ Departamento de Física de Materiales, Facultad de Físicas, Universidad Complutense, E-28040 Madrid, \\ Spain \\ V. G. Golubev, D. A. Kurdyukov, A. B. Pevtsov, and M. V. Zamoryanskaya \\ Ioffe Physico-Technical Institute, RAS, 194021 St. Petersburg, Russia
}

(Received 20 June 2000; accepted for publication 13 September 2000)

\begin{abstract}
Scanning tunneling microscopy and scanning tunneling spectroscopy (STS) are used to investigate the local electronic behavior of $\mathrm{Pt}-\mathrm{Si}$ nanostructures fabricated in an opal matrix formed by silica spheres of $250 \mathrm{~nm}$ diameter. Si and Pt are regularly distributed inside the opal pores and form nanoscale metal-semiconductor-metal junctions. Normalized differential conductance curves enable us to study the distribution of $\mathrm{Pt}$ and $\mathrm{Si}$ and to detect the presence of regions showing a surface band gap in the range $0.5-0.8 \mathrm{eV}$, possibly associated with the formation of silicides. STS appears as a suitable technique for the electrical characterization of opal-based nanostructures. (C) 2000 American Institute of Physics. [S0003-6951(00)02146-X]
\end{abstract}

Synthetic opals, which are composed of ordered amorphous $\mathrm{SiO}_{2}$ spheres forming a face centered cubic lattice, have attracted interest in the past years because of several potential applications of these systems in optoelectronics. Some applications relate to the photonic band gap phenomenon in opals, that has been investigated by different authors, e.g., Refs. 1-3. On the other hand, synthetic opal has a regular sublattice of submicron channels and voids permitting us to obtain three-dimensional (3D) assemblies of silicon nanostructures in opal matrices which can be used as the basis of objects for microelectronics, e.g., solid state devices with $p-n$ junctions or Schottky diodes. For the fabrication of opalbased silicon solid-state electronic devices it is necessary to deposit a homogeneous layer on the internal surface of the voids and to create metallic contacts. Feoktistov et al. ${ }^{4}$ have formed $\mathrm{Pt}-\mathrm{Si}$ contacts by coating the silica spheres with a platinum layer before embedding in silicon and demonstrated the possibility of creating a 3D multilayer semiconductor structures ( $p-n$ junctions or Schottky barriers) on the inner surface of the opal voids. These authors ${ }^{4,5}$ obtained 3D cluster systems with cluster sizes ranging from 1 to $100 \mathrm{~nm}$ and a density of elements as high as $10^{14} \mathrm{~cm}^{-3}$. In the present work we use scanning tunneling microscopy (STM) and a spatially resolved scanning tunneling spectroscopy (STS) technique, namely current imaging tunneling spectroscopy (CITS), ${ }^{6}$ to investigate the local electronic properties of the opal-Pt-Si samples used in Refs. 4 and 5. In particular, CITS measurements allow us to study the $I-V$ behavior and the band gaps in different places of the structure and to observe nanoscale active semiconductor elements.

The opals used in this work consist of $\mathrm{SiO}_{2}$ spheres of about 250-nm-diameter forming a face centered cubic lattice. The sizes of the octahedral and tetrahedral voids in this lattice are of about 100 and $50 \mathrm{~nm}$, respectively. For the preparation of the opal-platinum-silicon samples, the opals were

${ }^{a)}$ Electronic mail: piqueras@eucmax.sim.ucm.es embedded first with platinum and then with silicon following the procedure described in Ref. 4. The fill factors of Si and Pt evaluated from electron probe microanalysis results were $65 \%$ and $25 \%$, respectively. Transmission electron microscopy (TEM) has shown ${ }^{4}$ that on the surface of the silica spheres different distributions of platinum may result. In some cases, platinum particles in the range of several nanometers and in different stages of coalescence are observed, while it is also possible to obtain a uniform platinum layer of 5-6 nm thickness. In the samples used in this work the spheres have a discontinuous platinum distribution on their surface. These samples were cleaved and freshly exposed spheres close to the original surface were chosen for the observations. Such spheres are in the sample region that was filled with platinum and silicon.

The samples were observed in the secondary electron mode of a Leica 440 scanning electron microscope (SEM) operating under a vacuum of $1 \times 10^{-6}$ Torr. The STM used was a small size instrument fixed in the chamber of the SEM. Possible influence of surface contamination due to the SEM electron beam on the STM measurements was either minimized by using beam currents under $10 \mathrm{pA}$ for short time or avoided by performing STM observations in areas not previously observed with the SEM. The main features of this instrument are similar to the one previously described in Ref. 7. For SEM and STM observations the opals were glued with silver paint to the specimen holder. This provides good electrical contact to the samples, as shown by the absence of charge effects during SEM observations as well as by the good stability of the tunneling current during STM measurements. In addition, no surface drop of the applied tunneling voltage was found in the samples investigated. The STM was operated in the constant-current and CITS modes using electrochemically etched or mechanically sharpened Pt-Ir wires as probe tips. CITS provides real space imaging of surface electronic states by recording $I-V$ curves at fixed tip-sample separation at every pixel within an image. In addition to the $I-V$ curves, current images can be formed by plotting the 


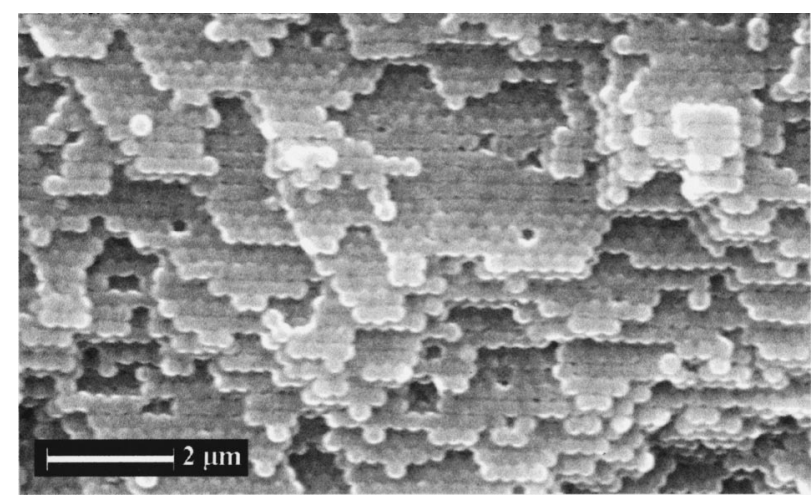

FIG. 1. SEM image of one of the opal samples investigated showing the distribution of the silica spheres.

measured current at any voltage. Details of the CITS procedure used here have been described elsewhere. ${ }^{8}$ In order to analyze the $I-V$ data of the different points of an image, the normalized differential conductance $(d I / d V) /(I / V)$ spectra were used. This quantity removes most of the exponential dependence of tunneling current on tip-sample separation ${ }^{9}$ and is proportional to the surface density of states. ${ }^{10,11}$ Measurements performed with different tips and under different tunneling conditions confirmed the reproducibility of the obtained results.

SEM images show the regular distribution of the $\mathrm{SiO}_{2}$ spheres with diameters of about $250 \mathrm{~nm}$ (Fig. 1). The spheres are also imaged in the constant current mode of the STM, which shows that they are conductive due to the platinum and silicon cover on their surface. Figure 2 is the STM image, obtained under an applied bias of $0.9 \mathrm{~V}$ and $0.3 \mathrm{nA}$ tunneling current, of the contact region of three spheres.

The $(d I / d V) /(I / V)$ curves recorded on the free surface of the spheres, like the region marked as 1 in Fig. 2, show a conduction behavior that depends on the point considered (Fig. 3). Two different kinds of curves are obtained; one represents a metallic conduction [curve (a)] and is related to the existence of platinum clusters. The other one, in which a surface band gap of about $1 \mathrm{eV}$ is observed, indicates the

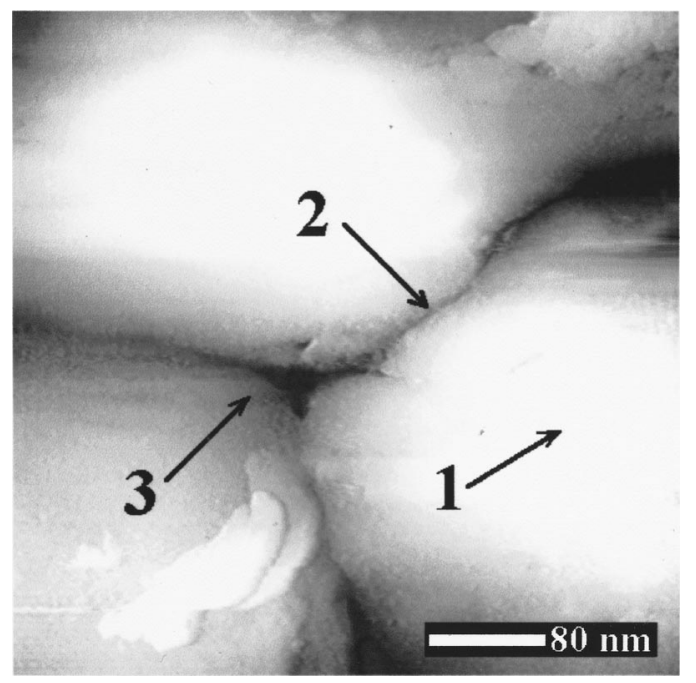

FIG. 2. Constant current STM image, acquired with a sample voltage of 0.9 $\mathrm{V}$ and $0.3 \mathrm{nA}$ tunneling current, showing the contact region of three spheres of an opal. Gray scale range is $49 \mathrm{~nm}$.

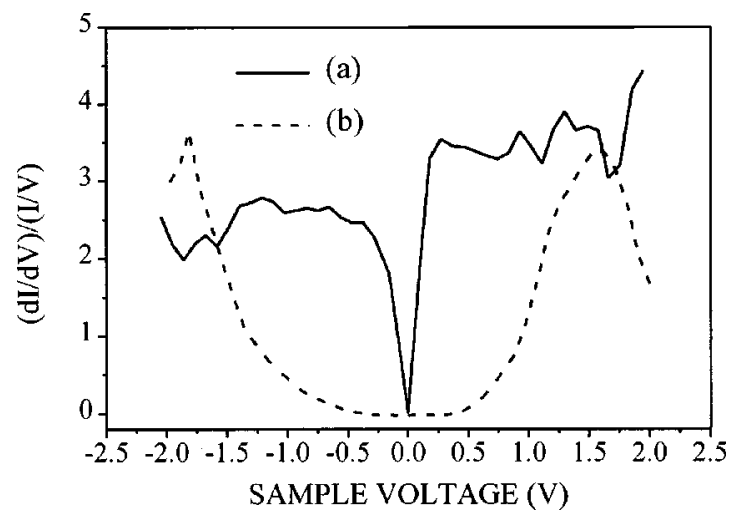

FIG. 3. The $(d I / d V) /(I / V)$ spectra usually recorded at regions like that labeled as 1 in Fig 2. (a) Curve showing metallic behavior related to the existence of $\mathrm{Pt}$ at the surface, (b) curve showing semiconducting behavior indicating the presence of $\mathrm{Si}$.

presence of silicon [curve (b)]. In the contact region of the spheres (labeled as 2 in Fig. 2) a more complex behavior has been observed. In addition to the curves attributed to platinum and silicon, as those shown in Fig. 3, other curves reveal the existence of surface band gaps in the range $0.5-0.8$ $\mathrm{eV}$. An example of this behavior is shown in Fig. 4. We tentatively explain this result by considering the different species detected in these regions by TEM. ${ }^{4,12}$ The experimentally measured interplanar distances on high-resolution images demonstrated the presence of platinum and silicon, but interplanar distances which could not be related to these elements were measured as well. By comparison of the unidentified interplanar distances with those of $\mathrm{Pt}-\mathrm{Si}$ compounds, close matchings were observed with the $\mathrm{Pt}_{12} \mathrm{Si}_{5}$ tetragonal phase and the PtSi hexagonal phase. The formation of the $\mathrm{Pt}_{12} \mathrm{Si}_{5}$ phase can take place during the thermal treatment of filled opal fabrication at temperatures at which this phase is formed from Pt deposited on silicon wafers. ${ }^{13,14} \mathrm{We}$ suggest that the band gaps in the range $0.5-0.8 \mathrm{eV}$, measured by tunneling spectroscopy in the area of contact between spheres, could be related to the presence of Pt silicides. This suggestion is also supported by theoretical calculations on the electronic structure of silicides, in which band gaps narrower than that corresponding to silicon were predicted. ${ }^{15}$ However, more data on the electronic structure of these compounds would be necessary to confirm this possibility.

Another specific feature of the conductance measure-

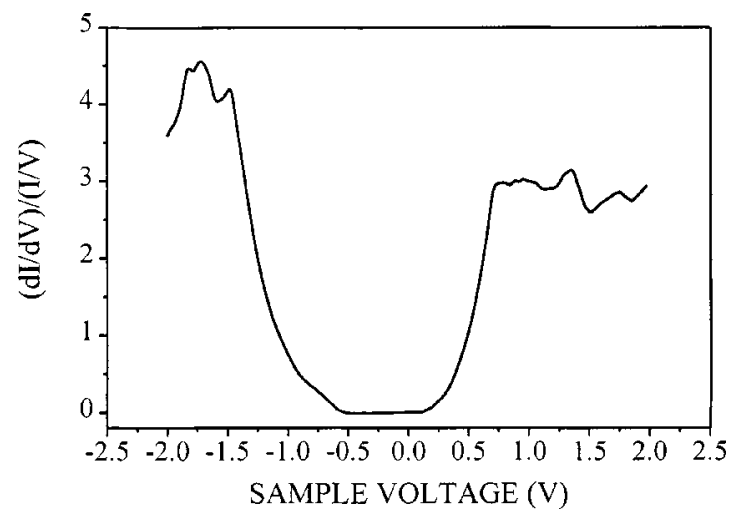

FIG. 4. Normalized differential conductance spectrum acquired at position labeled as 2 in Fig. 2. 


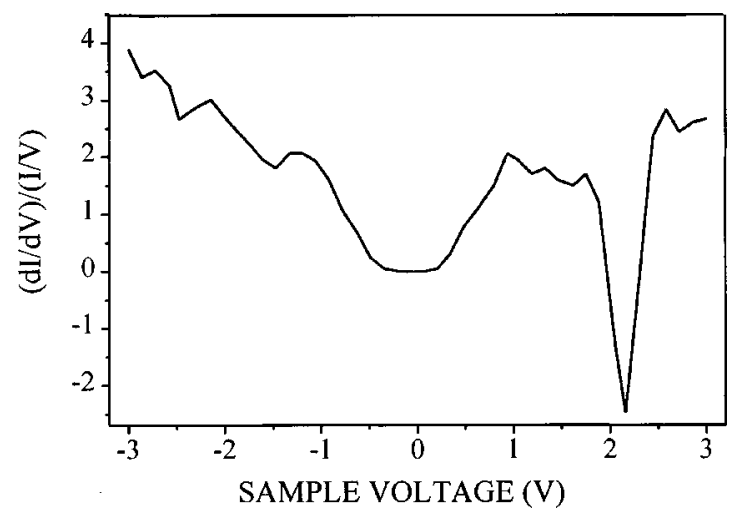

FIG. 5. The $(d I / d V) /(I / V)$ curve, showing negative differential conductance at about $+2.2 \mathrm{~V}$ sample voltage, recorded at the contact region of two spheres of a sample.

ments performed in these regions is the appearance in some of the curves (about $20 \%$ of the total analyzed) of negative differential conductance around $+2.2 \mathrm{~V}$ applied bias voltage (Fig. 5). This effect has been previously reported, e.g., Refs. 16 and 17, in tunneling spectroscopy investigations of ultrathin oxide films on silicon and one of the suggested mechanisms is related to an energy level matching between the tip Fermi level and a defect level, such as a dangling bond state positioned at the midgap of the oxide. A similar mechanism associated with the presence of $\mathrm{nm}$-sized $\mathrm{Si}$ clusters located at the inter-sphere region of the opals could be responsible for the negative conductance observed here.

The differences in the electronic properties of different regions of the sample, revealed in the $(d I / d V) /(I / V)$ curves, are also appreciated in the CITS images. Figure 6 shows a CITS image of an opal sample in which an enhanced contrast at the periphery of the spheres, related to local conductance variations, can be clearly observed.

In those areas like that marked as 3 in Fig. 2 the differential conductance curves show frequently the same metallic behavior shown in Fig. 3(a). This result agrees with the presence of bigger platinum particles observed by TEM in these regions.

In summary, a three-dimensional regular lattice of platinum-silicon nanostructures fabricated using an opal matrix has been characterized by scanning tunneling spectroscopy. The conductance behavior of the structure has been analyzed with high spatial resolution to determine the presence of $\mathrm{Pt}$ and $\mathrm{Si}$ in the different regions of the sample. The results indicate the formation of silicides in the contact areas of the silica spheres. Tunneling spectroscopy appears as a powerful tool to investigate the electronic behavior of such nanostructures.

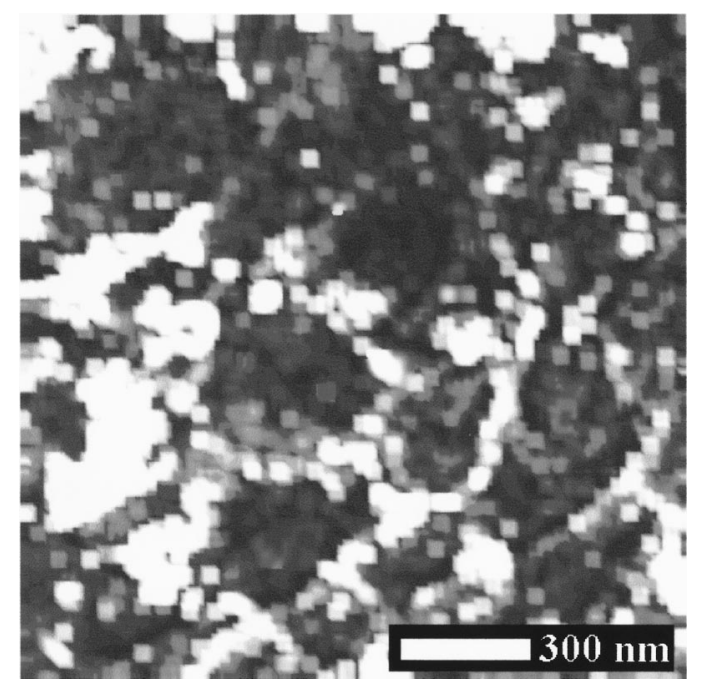

FIG. 6. CITS image acquired at $-1.75 \mathrm{~V}$ sample voltage in one of the opals. Gray scale range is $2.9 \mathrm{nA}$.

This work was supported by DGES (Project No. PB960639), the Russian R\&D program "Nanostructures" (Grant No. 97-2016) and RFBR under Grant No. 98-02-17350.

${ }^{1}$ Yu. A. Vlasov, V. N. Astratov, O. Z. Karimov, and A. A. Kaplyanskii, Phys. Rev. B 55, 13357 (1997).

${ }^{2}$ V. N. Bogomolov, S. V. Gaponenko, I. N. Germanenko, A. M. Kapitonov, E. P. Petrov, N. V. Gaponenko, A. V. Prokofiev, A. N. Ponyavina, N. I. Silvanovich, and S. M. Samoilovich, Phys. Rev. E 55, 7619 (1997).

${ }^{3}$ A. Blanco, C. López, R. Mayoral, H. Míguez, F. Meseguer, A. Mifsud, and J. Herrero, Appl. Phys. Lett. 73, 1781 (1998).

${ }^{4}$ N. A. Feoktistov, V. G. Golubev, J. L. Hutchison, D. A. Kurdyukov, A. B. Pevtsov, R. Schwarz, J. Sloan, and L. M. Sorokin, Mater. Res. Soc. Symp. Proc. 609, A24.4.1 (2000).

${ }^{5}$ V. N. Bogomolov, N. A. Feoktistov, V. G. Golubev, J. L. Hutchison, D. A. Kurdyukov, A. B. Pevtsov, R. Schwarz, J. Sloan, and L. M. Sorokin, J. Non-Cryst. Solids 266-269, 1021 (2000).

${ }^{6}$ R. J. Hamers, R. M. Tromp, and J. E. Demuth, Phys. Rev. Lett. 56, 1972 (1986).

${ }^{7}$ A. Asenjo, A. Buendía, J. M. Gómez-Rodríguez, and A. Baró, J. Vac. Sci. Technol. B 12, 1658 (1994).

${ }^{8}$ G. Panin, C. Díaz-Guerra, and J. Piqueras, Appl. Phys. Lett. 72, 2129 (1998).

${ }^{9}$ J. A. Stroscio, R. M. Feenstra, and A. P. Fein, Phys. Rev. Lett. 57, 2579 (1986).

${ }^{10}$ N. D. Lang, Phys. Rev. B 34, 5947 (1986).

${ }^{11}$ R. M. Feenstra, J. A. Stroscio, and A. P. Fein, Surf. Sci. 181, 295 (1987).

${ }^{12}$ N. A. Feoktistov, V. G. Golubev, D. A. Kurdyukov, A. B. Pevtsov, V. V. Ratnikov, L. M. Sorokin, J. L. Hutchison, and J. Sloan (unpublished results).

${ }^{13}$ B. Tsui and M. Chen, J. Appl. Phys. 68, 6246 (1990).

${ }^{14}$ S. Jin, H. Bender, R. A. Donaton, and K. Maex, Inst. Phys. Conf. Ser. 157, 497 (1997).

${ }^{15}$ O. Bisi and C. Calandra, J. Phys. C 14, 5479 (1981).

${ }^{16}$ M. Tabe and M. Tanimoto, Appl. Phys. Lett. 58, 2105 (1991).

${ }^{17}$ H. Watanabe, K. Fujita, and M. Ichikawa, Appl. Phys. Lett. 72, 1987 (1998). 